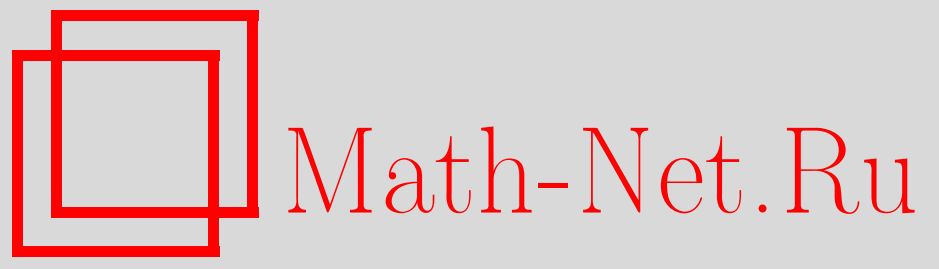

О. Ш. Шарипов, Ограниченный закон повторного логарифма для слабозависимых случайных величин со значениями в банаховых пространствах типа 2, Теория вероятн. и ее примен., 2004, том 49, выпуск $3,522-537$

DOI: https://doi.org/10.4213/tvp206

Использование Общероссийского математического портала Math-Net.Ru подразумевает, что вы прочитали и согласны с пользовательским соглашением

http: //www.mathnet.ru/rus/agreement

Параметры загрузки:

IP : 3.80 .253 .173

26 апреля 2023 г., 15:57:56






\section{ОГРАНИЧЕННЫЙ ЗАКОН ПОВТОРНОГО ЛОГАРИФМА ДЛЯ СЛАБОЗАВИСИМЫХ СЛУЧАЙНЫХ ВЕЛИЧИН СО ЗНАЧЕНИЯМИ В БАНАХОВЫХ ПРОСТРАНСТВАХ ТИПА $2^{1)}$}

В статье рассматривается последовательность одинаково распределенных случайных величин с $\psi$-перемешиванием и со значениями в банаховом пространстве типа 2, норма которого удовлетворяет определенным условиям. Для этой последовательности при оптимальных моментных условиях доказывается ограниченный закон повторного логарифма.

Ключевые слова и фразы: ограниченный закон повторного логарифма, $\psi$-перемешивание, банахово пространство типа 2.

1. Введение и формулировка результата. Закон повторного логарифма в различных постановках изучался многими авторами (см. [1]-[17]). В настоящей работе мы рассмотрим ограниченный закон повторного логарифма (ОЗПЛ). Пусть $\left\{X_{n}, n \geqslant 1\right\}$ - последовательность центрированных случайных величин (с.в.), принимающих значения в банаховом пространстве $B$ с нормой $\|\cdot\|$. Мы будем говорить, что $\left\{X_{n}, n \geqslant 1\right\}$ удовлетворяет ОЗПЛ, если

$$
\limsup _{n \rightarrow \infty} \frac{\left\|X_{1}+\cdots+X_{n}\right\|}{\sqrt{2 n \ln \ln n}}<\infty \quad \text { п.н. }
$$

Мы не будем делать обзор результатов и методов упомянутых выше работ, но вкратце скажем о работах, непосредственно относящихся к настоящей статье. Для последовательностей независимых одинаково распределенных $B$-значных с.в. необходимые и достаточные условия для ОЗПЛ получены в [1]-[3]. В работах [5]-[6] ОЗПЛ доказан для последовательностей, удовлетворяющих некоторым условиям перемешивания. В этих работах предполагается выполнение условия $\mathbf{E}\left\|X_{k}\right\|^{2+\delta}<\infty$, $k=1,2, \ldots$, для некоторого $\delta>0$. Из результатов работы [9] можно получить ОЗПЛ при условии $\mathbf{E}\left\|X_{1}\right\|^{2}<\infty$ для стационарной последовательности $\mathbf{R}^{k}$-значных с.в., удовлетворяющих определенным условиям перемешивания. В [17] получено необходимое и достаточное моментное

* Институт математики им. В.И. Романовского АН УЗ, ул. Ф. Ходжаева, 29, 700125 Ташкент, Узбекистан; e-mail: mathinst@uzsci.net 
условие для стационарной последовательности гильбертовозначных с.в., удовлетворяющих условию равномерно сильного перемешивания. Необходимые и достаточные условия выполнимости ОЗПЛ для стационарной в узком смысле последовательности $m$-зависимых с.в. со значениями в $B$ получены в [18].

В настоящей работе мы приведем аналогичный результат для случая банаховых пространств типа 2, норма которых удовлетворяют определенным условиям. Напомним, что $B$ имеет тип 2 , если для любого набора независимых $B$-значных с.в. $X_{1}, \ldots, X_{n}$ с $\mathbf{E} X_{k}=0, \mathbf{E}\left\|X_{k}\right\|^{2}<\infty$, $k=1, \ldots, n$, выполнено неравенство

$$
\mathbf{E}\left\|\sum_{k=1}^{n} X_{k}\right\|^{2} \leqslant C \sum_{k=1}^{n} \mathbf{E}\left\|X_{k}\right\|^{2}
$$

где константа $C$ зависит только от пространства $B$.

В дальнейшем $B$ будет означать банахово пространство типа 2 , вторая производная (по направлению) нормы $D_{x}^{2}$ которого удовлетворяет 1) условию $\sup _{\|x\|=1}\left\|D_{x}^{2}\right\|_{*}<\infty$, где $\|\cdot\|_{*}$ означает норму второй производной, и 2) условию Гёльдера со степенью $\alpha$ вне точки 0 .

Здесь и в дальнейшем нулевые элементы рассматриваемых пространств также будем обозначать через 0 .

Определение производной по направлению и примеры пространств, норма которых удовлетворяет приведенным выше условиям, можно найти в [19]. Отметим, что пространства $L_{p}, p \geqslant 2$, удовлетворяют этим условиям.

Для последовательности $\left\{X_{n}, n \geqslant 1\right\}$ определим коэффициент $\psi$-перемешивания:

$$
\psi(n)=\sup _{k, A \in \mathscr{F}_{1}^{k}, B \in \mathscr{F}_{k+n}^{\infty}} \frac{|\mathbf{P}(A B)-\mathbf{P}(A) \mathbf{P}(B)|}{\mathbf{P}(A) \mathbf{P}(B)},
$$

где $\mathscr{F}_{k}^{n}-\sigma$-алгебра, порожденная с.в. $X_{k}, X_{k+1}, \ldots, X_{n}$.

Обозначим $S_{n}=X_{1}+\cdots+X_{n}, B^{*}-$ сопряженное пространство (норму которого также обозначим $\|\cdot\|), L x=\max (1, \ln x), \alpha_{n}=$ $\sqrt{2 n \ln \ln n}, n \geqslant 3$.

Основным результатом является следующая теорема.

Теорема. Пусть $\left\{X_{n}, n \geqslant 1\right\}-$ последовательность одинаково распределенных с.в. со значениями в $B$, для которой выполнены следующие условия:

$$
\begin{gathered}
\mathbf{E} X_{1}=0, \quad \mathbf{E} f^{2}\left(X_{1}\right)<\infty \quad \text { для всех } f \in B^{*} \\
\sum_{k=1}^{\infty} \psi(k)<\infty, \\
\lim _{n \rightarrow \infty} \frac{\mathbf{E} f^{2}\left(S_{n}\right)}{n}=\sigma_{t}<\infty \quad \text { для всех } f \in B^{*}
\end{gathered}
$$


Тогда для того, итобы имело место (1), необходимо и достаточно, чтобобl

$$
\mathbf{E} \frac{\left\|X_{1}\right\|^{2}}{L L\left\|X_{1}\right\|}<\infty
$$

Заметим, что для независимых одинаково распределенных с.в. со значениями в банаховом пространстве типа 2 необходимым и достаточным условием ОЗПЛ являются (2) и (5) (см. [3]).

Если $\left\{X_{n}, n \geqslant 1\right\}$ стационарна в узком смысле, то условие (4) излишне (см. [20, теорема 4.1]). Также заметим, что теорема остается верной и для $\left\{X_{n}, n \geqslant 1\right\}$ с равномерно сильным перемешиванием, если для таковых выполнено неравенство (А), так как все остальные используемые при доказательстве факты имеют место и для последовательности с равномерно сильным перемешиванием. ОЗПЛ и компактный закон повторного логарифма для последовательностей гильбертовозначных с.в. с равномерно сильным перемешиванием доказаны в [21], [22].

Разумеется, (4) выполняется не только для стационарных в узком смысле последовательностей. Например, (4) имеет место для слабо стационарных последовательностей (см. [5], [6]), но при более ограничительных условиях на коэффициенты перемешивания.

2. Доказательство теоремы. Д о с т а т о ч н о с т ь. Всюду в дальнейшем буквой $C$ будем обозначать абсолютные и не абсолютные константы, возможно разные даже в одной цепочке неравенств. $C$ может зависеть от разных параметров, но не зависит от индексов суммирования и от числа слагаемых (не зависит от $n, p, q, m, r, k, i, j$ и т.д.). Необходимые утверждения мы будем формулировать в виде лемм. Ради удобства они приводятся непосредственно перед их применением.

Пусть $\beta>1$. Введем следующие обозначения:

$$
n_{r}=\left[\beta^{r}\right], \quad I(r)=\left\{n_{r}+1, \ldots, n_{r+1}\right\}, \quad \tau_{r}=2 n_{r+1} L L n_{r+1}=\alpha_{n_{r+1}}^{2},
$$

$[k]$ - целая часть $k$. Запись $a_{n} \sim b_{n}$ означает существование абсолютных констант $c_{1}>0, c_{2}>0$ таких, что $c_{1}<a_{n} / b_{n}<c_{2}, n=1,2, \ldots$

Лемма 1 (см. [1]). Пусть $\mu$ - вероятностная мера на $B$ с ковариачионной функиией $T(f, g)$. Рассмотрим отображение $S: B^{*} \rightarrow B$, определенное следуюшим образом:

$$
S f=\int_{B} x f(x) d \mu .
$$

Здесь интеграл понимается в смысле Петтиса. Обозначим через $H_{\mu}$ замыккание образа $S$ и в $H_{\mu}$ введем норму с помощью следующего скалярного произведения:

$$
\langle S f, S g\rangle=\int_{B} f(x) g(x) d \mu .
$$


Тогда, если $K$ - единичный шар $H_{\mu}$, то $K$ будет замкнутыцм, симметричным, выпукльм множеством в $B$ и для всех $f \in B^{*}$

$$
\sup _{x \in K} f(x)=\left(\int_{B} f^{2}(y) d \mu\right)^{1 / 2} .
$$

Существование

$$
T(f, g)=\lim _{n \rightarrow \infty} \frac{\mathbf{E} f\left(S_{n}\right) g\left(S_{n}\right)}{n}
$$

следует из (4).

Произведем срезку следующим образом;

$$
\begin{array}{cc}
u_{j}=X_{j} I\left(\left\|X_{j}\right\|^{2} \leqslant \tau_{r}\right)-\mathbf{E} X_{j} I\left(\left\|X_{j}\right\|^{2} \leqslant \tau_{r}\right), & j \in I(r), \\
w_{j}=X_{j} I\left(\left\|X_{j}\right\|^{2}>\tau_{r}\right)-\mathbf{E} X_{j} I\left(\left\|X_{j}\right\|^{2}>\tau_{r}\right), & j \in I(r), \\
U_{n}=\sum_{j=1}^{n} u_{j}, \quad W_{n}=\sum_{j=1}^{n} w_{j} . &
\end{array}
$$

В определениях $u_{j}$ и $w_{j} I(A)$ ес'гь индикатор события $A$.

Положим $\Gamma=\sup _{x \in K}\|x\|=\sup _{\|f\| \leqslant 1} T^{1 / 2}(f, f)$.

Заметим, что достаточно доказать утверждение теоремы в предположении $\Gamma>0$. В случае $\Gamma=0$ утверждение теоремы доказывается точно так же, как в [18, с. 712] (см. также [21]). По этой причине в дальнейшем предполагается, что $\Gamma>0$.

Нам надо доказать, что для всех $\varepsilon>0$

$$
\begin{aligned}
& \limsup _{n \rightarrow \infty} \frac{\left\|U_{n}\right\|}{\alpha_{n}} \leqslant \Gamma+\varepsilon \quad \text { п.н., } \\
& \limsup _{n \rightarrow \infty} \frac{\left\|W_{n}\right\|}{\alpha_{n}}=0 \quad \text { п.н., }
\end{aligned}
$$

откуда в силу произвольности $\varepsilon$ будет следовать, что

$$
\limsup _{n \rightarrow \infty} \frac{\left\|S_{n}\right\|}{\alpha_{n}} \leqslant \Gamma \quad \text { п.н. }
$$

Лемма 2. Если выполнено (5), то имеет место (7).

Эта лемма доказана А. де Акостой и Дж. Куэлбсом в [2] для независимого случая, но без использования свойств независимости. Поэтому в нашем случае доказательство сохраняется (см. также [21]).

Докажем (6). Покажем, что существует достаточно близкое к единице $\beta>1$ такое, что

$$
\limsup _{r \rightarrow \infty} \max _{n \in I(r)}\left\|\frac{U_{n}}{\alpha_{n}}\right\| \leqslant \Gamma+\varepsilon \quad \text { п.н. }
$$


В силу возрастания $\alpha_{n}$ имеет место следующее неравенство:

$$
\max _{n \in I(r)}\left\|\frac{U_{n}}{\alpha_{n}}\right\| \leqslant \frac{\alpha_{n_{r+1}}}{\alpha_{n_{r}}} \max _{n \in I(r)}\left\|\frac{U_{n}}{\alpha_{n_{r+1}}}\right\| \text { п.н. }
$$

Из (9) и соотношения $\alpha_{n_{r+1}} / \alpha_{n_{r}} \sim \sqrt{\beta}$ имеем

$$
\limsup \max _{r \rightarrow \infty} \frac{\left\|U_{n}\right\|}{\alpha_{n}} \leqslant \sqrt{\beta} \limsup \max _{r \rightarrow \infty} \frac{\left\|U_{n}\right\|}{\alpha_{n \in I(r)}} \text { п.н. }
$$

Так как мы можем выбрать $\beta$ сколь угодно близким к 1 , то для доказательства (8) достаточно показать, что

$$
\limsup _{r \rightarrow \infty} \max _{n \in I(r)} \frac{\left\|U_{n}\right\|}{\alpha_{n_{r+1}}} \leqslant \Gamma+\varepsilon \quad \text { п.н. }
$$

Произведем секционирование следующим образом. Зафиксировав $n \in I(r)$, положим

$$
\xi_{i}=\sum_{j=(p+q)(i-1)+1}^{(p+q)(i-1)+p} u_{j}, \quad \eta_{i}=\sum_{j=(p+q) i-q+1}^{(p+q) i} u_{j} .
$$

Для $n \in I(r)$ имеем

$$
U_{n}=\sum_{i=1}^{m} \xi_{i}+\sum_{i=1}^{k} \eta_{i}+U_{0}
$$

где $U_{0}$ есть «остаток», остающийся после секционирования $\xi_{1}, \eta_{1}, \xi_{2}, \eta_{2}, \ldots$ до тех пор пока для очередного $\xi$ или $\eta$ не будет достаточного количества $X_{i}$. Заметим, что $m=m(n), k=k(n),|m-k| \leqslant 1$. Выбираем

$$
p=\left[\frac{\beta^{r}}{r^{\gamma}}\right], \quad \gamma>\max \left(1, \frac{2}{\alpha}\right), \quad q=\left[\beta^{\alpha_{0} r}\right], \quad 0<\alpha_{0}<1 .
$$

Тогда $m \sim k \sim r^{\gamma}$.

Далее, имеем:

$$
\begin{aligned}
& \limsup \max _{r \rightarrow \infty}\left\|\frac{U_{n}}{\alpha_{n_{r+1}}}\right\| \leqslant \limsup \max _{r \rightarrow \infty}\left\|\frac{\sum_{i=1}^{m} \xi_{i}}{\alpha_{n_{r+1}}}\right\| \\
& +\limsup _{r \rightarrow \infty} \max _{n \in I(r)}\left\|\frac{\sum_{i=1}^{k} \eta_{i}}{\alpha_{n_{r+1}}}\right\|+\limsup _{r \rightarrow \infty} \max _{n \in I(r)}\left\|\frac{U_{0}}{\alpha_{n_{r+1}}}\right\| \text { п.н. }
\end{aligned}
$$

Покажем, что два последних слагаемых в правой части неравенства (12) п.н. равны нулю. Приведем лемму, которую будем часто использовать.

Лемма 3 (см. [23]). Пусть $\left\{X_{n}, n \geqslant 1\right\}$ - последовательность чентрированньх $B$-значных с.в., удовлетворяюшая условию (3). Тогда для $t \geqslant 2$ имеет место следующее неравенство:

$$
\mathbf{E} \max _{1 \leqslant k \leqslant n}\left\|S_{k}\right\|^{t} \leqslant C\left(\sum_{k=1}^{n} \mathbf{E}\left\|X_{k}\right\|^{t}+\left(\mathbf{E}\left\|S_{n}\right\|^{2}\right)^{t / 2}\right) .
$$


В силу теоремы 1 из [24], где при условии (3) доказано неравенство

$$
\mathbf{E}\left\|S_{n}\right\|^{2} \leqslant C \sum_{k=1}^{n} \mathbf{E}\left\|X_{k}\right\|^{2}
$$

имеем

$$
\mathbf{E} \max _{1 \leqslant k \leqslant n}\left\|S_{k}\right\|^{t} \leqslant C\left(\sum_{k=1}^{n} \mathbf{E}\left\|X_{k}\right\|^{t}+\left(\sum_{k=1}^{n} \mathbf{E}\left\|X_{k}\right\|^{2}\right)^{t / 2}\right) .
$$

Применяя (13) и следующее простое неравенство:

$$
\mathbf{E}\left\|X_{1} I\left(\left\|X_{1}\right\|^{2} \leqslant \tau_{r}\right)-\mathbf{E} X_{1} I\left(\left\|X_{1}\right\|^{2} \leqslant \tau_{r}\right)\right\|^{2} \leqslant 4 \mathbf{E}\left\|X_{1}\right\|^{2} I\left(\left\|X_{1}\right\|^{2} \leqslant \tau_{r}\right),
$$

получим

$$
\begin{aligned}
& \sum_{r=1}^{\infty} \mathbf{P}\left\{\max _{n \in I(r)}\left\|\frac{U_{0}}{\alpha_{n_{r+1}}}\right\|>\varepsilon\right\} \leqslant \sum_{r=1}^{\infty} \frac{\mathbf{E} \max _{n \in I(r)}\left\|U_{0}\right\|^{2}}{\varepsilon^{2} \alpha_{n_{r+1}}^{2}} \\
& \leqslant \sum_{r=1}^{\infty} \frac{C p \mathbf{E}\left(\left\|X_{1}\right\|^{2} I\left(\left\|X_{1}\right\|^{2} \leqslant \tau_{r}\right)\right)}{\varepsilon^{2} \alpha_{n_{r+1}}^{2}} \\
& \leqslant \sum_{r=1}^{\infty} \frac{C p}{\varepsilon^{2} \beta^{r}} \mathbf{E}\left(\frac{\left\|X_{1}\right\|^{2}}{L L\left\|X_{1}\right\|} I\left(\left\|X_{1}\right\|^{2} \leqslant \tau_{r}\right) \frac{L L\left\|X_{1}\right\|}{L L \tau_{r}}\right), \\
& \sum_{r=1}^{\infty} \mathbf{P}\left\{\max _{n \in I(r)}\left\|\frac{\sum_{i=1}^{k} \eta_{i}}{\alpha_{n_{r+1}}}\right\|>\varepsilon\right\} \leqslant \sum_{r=1}^{\infty} \frac{\mathbf{E}_{\max _{n \in I(r)}\left\|\sum_{i=1}^{k} \eta_{i}\right\|^{2}}}{\varepsilon^{2} \alpha_{n_{r+1}}^{2}} \\
& \leqslant \sum_{r=1}^{\infty} \frac{C k q}{\varepsilon^{2} \alpha_{n_{r+1}}^{2}} \mathbf{E}\left(\left\|X_{1}\right\|^{2} I\left(\left\|X_{1}\right\|^{2} \leqslant \tau_{r}\right)\right) \\
& \leqslant \sum_{r=1}^{\infty} \frac{C k q}{\varepsilon^{2} \beta^{r}} \mathbf{E}\left(\frac{\left\|X_{1}\right\|^{2}}{L L\left\|X_{1}\right\|} I\left(\left\|X_{1}\right\|^{2} \leqslant \tau_{r}\right) \frac{L L\left\|X_{1}\right\|}{L L \tau_{r}}\right) .
\end{aligned}
$$

Из (11) вытекает сходимость двух последних рядов, следовательно, по лемме Бореля-Кантелли два последних слагаемых в правой части (12) п.н. равны нулю.

Для того чтобы оценить первое слагаемое в правой части неравенства (12), сначала получим следующую оценку:

$$
\begin{aligned}
\sup _{n \in I(r)} \mathbf{P} & \left\{\left\|\sum_{i=1}^{m(n)} \xi_{i}-\sum_{i=1}^{m\left(\left[\beta^{r+1}\right]\right)} \xi_{i}\right\|>\frac{\varepsilon}{2} \alpha_{n_{r+1}}\right\} \\
& \leqslant \sup _{n \in I(r)} \mathbf{P}\left\{\left\|\sum_{i=m(n)+1}^{m\left(\left[\beta^{r+1}\right]\right)} \xi_{i}\right\|>\frac{\varepsilon}{2} \alpha_{n_{r+1}}\right\} \\
& \leqslant C \frac{\left(\left[\beta^{r+1} / p\right]-\left[\beta^{r} / p\right]\right)^{1 / 2}}{\alpha_{n_{r+1}}} \sup _{n \in I(r)} \max _{m(n)<i \leqslant m\left(\left[\beta^{r+1}\right]\right)}\left(\mathbf{E}\left\|\xi_{i}\right\|^{2}\right)^{1 / 2} \\
& \leqslant C \frac{r^{\gamma / 2} p^{1 / 2}}{\alpha_{n_{r+1}}}\left(\mathbf{E}\left\|X_{1} I\left(\left\|X_{1}\right\|^{2} \leqslant \tau_{r}\right)-\mathbf{E} X_{1} I\left(\left\|X_{1}\right\|^{2} \leqslant \tau_{r}\right)\right\|^{2}\right)^{1 / 2}
\end{aligned}
$$




$$
\begin{aligned}
& \leqslant C \frac{\beta^{r / 2} L L \tau_{r}}{\alpha_{n_{r+1}}}\left\{\mathbf{E}\left(\frac{\left\|X_{1}\right\|^{2}}{L L\left\|X_{1}\right\|} I\left(\left\|X_{1}\right\|^{2} \leqslant \tau_{r}\right) \frac{L L\left\|X_{1}\right\|}{L L \tau_{r}}\right)\right\}^{1 / 2} \\
& \leqslant C\left\{\mathbf{E}\left(\frac{\left\|X_{1}\right\|^{2}}{L L\left\|X_{1}\right\|} I\left(\left\|X_{1}\right\|^{2} \leqslant \tau_{r}\right) \frac{L L\left\|X_{1}\right\|}{L L \tau_{r}}\right)\right\}^{1 / 2} .
\end{aligned}
$$

Последняя величина при $r \rightarrow \infty$ стремится к нулю.

Теперь используем следующую лемму.

Лемма 4 (см. [25]). Пусть $\left\{X_{n}, n \geqslant 1\right\}$ - последовательность с.в. со значениями в $B$, для которой

$$
\min _{1 \leqslant j \leqslant n} \mathbf{P}\left\{\left\|S_{n}-S_{j}\right\| \leqslant a\right\}-\psi(1)>0
$$

для некоторого $a>0$.

Тогда для всех $x$ имеет место следующее неравенство:

$$
\mathbf{P}\left\{\max _{1 \leqslant k \leqslant n}\left\|S_{k}\right\|>x\right\} \leqslant\left(\min _{1 \leqslant j \leqslant n} \mathbf{P}\left\{\left\|S_{n}-S_{j}\right\| \leqslant a\right\}-\psi(1)\right)^{-1} \mathbf{P}\left\{\left\|S_{n}\right\|>x-a\right\} .
$$

Лемма 4 доказана для одномерных с.в., но доказательство сохраняется и в случае банаховых пространств.

Заметим, что (14) влечет выполнение условия леммы 4 (с $a=$ $\left.\varepsilon \alpha_{n_{r+1}} / 2\right)$, так как для достаточно больших $n$ справедливо неравенство $\psi(q)<1$. Используя лемму 4 , получим

$$
\mathbf{P}\left\{\max _{n \in I(r)}\left\|\sum_{i=1}^{m(n)} \xi_{i}\right\|>(\Gamma+\varepsilon) \alpha_{n_{r+1}}\right\} \leqslant C \mathbf{P}\left\{\left\|\sum_{i=1}^{m\left(\left[\beta^{r+1}\right]\right)} \xi_{i}\right\|>\left(\Gamma+\frac{\varepsilon}{2}\right) \alpha_{n_{r+1}}\right\} .
$$

Дальнейшая наша цель - показать, что

$$
\sum_{r=1}^{\infty} \mathbf{P}\left\{\left\|\sum_{i=1}^{m\left(\left[\beta^{r+1}\right]\right)} \xi_{i}\right\|>\left(\Gamma+\frac{\varepsilon}{2}\right) \alpha_{n_{r+1}}\right\}<\infty
$$

По теореме Беркеша-Филиппа [7] существует последовательность независимых с.в. $\left\{y_{i}\right\}$ в $B$ таких, что $y_{i}$ и $\xi_{i}$ одинаково распределены и

$$
\mathbf{P}\left\{\left\|\xi_{i}-y_{i}\right\|>6 \psi(q)\right\} \leqslant 6 \psi(q), \quad i=1,2, \ldots .
$$

Так как из (3) и (12) вытекает, что

$$
\sum_{r=1}^{\infty} \mathbf{P}\left\{\left\|\sum_{i=1}^{m\left(\left[\beta^{r+1}\right]\right)}\left(\xi_{i}-y_{i}\right)\right\|>\frac{\varepsilon}{4} \alpha_{n_{r+1}}\right\} \leqslant \sum_{r=1}^{\infty} C m\left(\left[\beta^{r+1}\right]\right) \psi(q)<\infty
$$

то нам остается доказать, что

$$
\sum_{r=1}^{\infty} \mathbf{P}\left\{\left\|\sum_{i=1}^{m\left(\left[\beta^{r+1}\right]\right)} y_{i}\right\|>\left(\Gamma+\frac{\varepsilon}{4}\right) \alpha_{n_{r+1}}\right\}<\infty .
$$

Воспользуемся следующей леммой. 
Лемма 5 (см. [2]). Пусть $\left\{Z_{i}\right\}$ - последовательность независимых предгауссовских с.в. со значениями в $B$. Тогда имеет место неравенство

$$
\left|\mathbf{E} g\left(\sum_{i=1}^{n} Z_{i}\right)-\mathbf{E} g\left(\sum_{i=1}^{n} G_{i}\right)\right|<C(\delta, \Lambda, \alpha) \sum_{i=1}^{n}\left\|Z_{i}\right\|^{2+\alpha},
$$

где $\left\{G_{i}\right\}$ - последовательность независимых гауссовских с.в. в В таких, что $G_{i}$ имеет тот же ковариачионный оператор, что и $Z_{i}$,

$$
\begin{gathered}
g(x)=\Phi(\|x\|), \\
\Phi(t)= \begin{cases}0, & 0 \leqslant t \leqslant \Lambda, \\
\text { возрастает, } & \Lambda \leqslant t \leqslant \Lambda+\delta, \quad \Lambda>0, \quad \delta>0, \\
1, & t \geqslant \Lambda+\delta,\end{cases}
\end{gathered}
$$

$C(\delta, \Lambda, \alpha)$ - константа, зависящая только от $\delta, \Lambda, \alpha$ и не зависящая om $\left\{Z_{i}\right\} u\left\{G_{i}\right\}$.

Напомним, что случайный элемент $Z$ называется предгауссовским, если существует гауссовский элемент с таким же ковариационным оператором, что и у $Z$.

Так как $\left\{y_{i}\right\}$ удовлетворяет условиям леммы 5 , то, выбирая $\Lambda=$ $\Gamma+\varepsilon / 8, \delta=\varepsilon / 8$, получим:

$$
\begin{aligned}
& \mathbf{P}\left\{\left\|\sum_{i=1}^{m\left(\left[\beta^{r+1}\right]\right)} y_{i}\right\|>\left(\Gamma+\frac{\varepsilon}{4}\right) \alpha_{n_{r+1}}\right\} \leqslant \mathbf{E} g\left(\sum_{i=1}^{m\left(\left[\beta^{r+1}\right]\right)} \frac{y_{i}}{\alpha_{n_{r+1}}}\right) \\
& \quad \leqslant \mathbf{E} g\left(\sum_{i=1}^{m\left(\left[\beta^{r+1}\right]\right)} \frac{G_{i}}{\alpha_{n_{r+1}}}\right)+C \sum_{i=1}^{m\left(\left[\beta^{r+1}\right]\right)} \frac{\mathbf{E}\left\|y_{i}\right\|^{2+\alpha}}{\alpha_{n_{r+1}}} .
\end{aligned}
$$

Подставляя (16) в ряд (15), оценим второе слагаемое с помощью (13):

$$
\begin{aligned}
& \sum_{r=1}^{\infty} \sum_{i=1}^{m\left(\left[\beta^{r+1}\right]\right)} \frac{\mathbf{E}\left\|y_{i}\right\|^{2+\alpha}}{\alpha_{n_{r+1}}}=\sum_{r=1}^{\infty} \sum_{i=1}^{m\left(\left[\beta^{r+1}\right]\right)} \frac{\mathbf{E}\left\|\xi_{i}\right\|^{2+\alpha}}{\alpha_{n_{r+1}}} \\
& \leqslant \sum_{r=1}^{\infty} \sum_{i=1}^{m\left(\left(\beta^{r+1}\right]\right)} \frac{C}{\alpha_{n_{r+1}}^{2+\alpha}}\left(\sum_{j=(p+q)(i-1)+1}^{(p+q)(i-1)+p} \mathbf{E} \| X_{j} I\left(\left\|X_{j}\right\|^{2} \leqslant \tau_{r}\right)\right. \\
&+\left(\sum_{j=(p+q)(i-1)+1}^{(p+q)(i-1)+p} \mathbf{E} X_{j} I\left(\left\|X_{j}\right\|^{2} \leqslant \tau_{r}\right) \|^{2+\alpha}\right. \\
& \\
&=I+I I .
\end{aligned}
$$


Оценим каждое слагаемое в отдельности. Имеем:

$$
\begin{aligned}
& I I=\sum_{r=1}^{\infty} \sum_{i=1}^{m\left(\left[\beta^{r+1}\right]\right)} \frac{C}{\alpha_{n_{r+1}}^{2+\alpha}}\left(\sum_{j=(p+q)(i-1)+1}^{(p+q)(i-1)+p} \mathbf{E} \| X_{j} I\left(\left\|X_{j}\right\|^{2} \leqslant \tau_{r}\right)\right. \\
& \left.-\mathbf{E} X_{j} I\left(\left\|X_{j}\right\|^{2} \leqslant \tau_{r}\right) \|^{2}\right)^{(2+\alpha) / 2} \\
& \leqslant \sum_{r=1}^{\infty} \sum_{i=1}^{m\left(\left[\beta^{r+1}\right]\right)} \frac{C}{\alpha_{n_{r+1}}^{2+\alpha}} p^{(2+\alpha) / 2}\left(\mathbf{E}\left(\left\|X_{1}\right\|^{2} I\left(\left\|X_{1}\right\|^{2} \leqslant \tau_{r}\right)\right)\right)^{(2+\alpha) / 2} \\
& \leqslant \sum_{r=1}^{\infty} \frac{C \beta^{r+1} p^{(2+\alpha) / 2}}{p \alpha_{n_{r+1}}^{2+\alpha}}\left(\mathbf{E}\left(\left\|X_{1} I\left(\left\|X_{1}\right\|^{2} \leqslant \tau_{r}\right)\right\|\right)\right)^{(2+\alpha) / 2} \\
& \leqslant \sum_{r=1}^{\infty}\left[\frac{C \beta^{r+1} p^{\alpha / 2}\left(L L \tau_{r}\right)^{(2+\alpha) / 2}}{\beta^{(r+1)(r+\alpha) / 2}\left(L L \beta^{r+1}\right)^{(2+\alpha) / 2}}\right. \\
& \left.\times\left(\mathbf{E}\left(\frac{\left\|X_{1}\right\|^{2}}{L L\left\|X_{1}\right\|} I\left(\left\|X_{1}\right\|^{2} \leqslant \tau_{r}\right) \frac{L L\left\|X_{1}\right\|}{L L \tau_{r}}\right)\right)^{(2+\alpha) / 2}\right] \\
& \leqslant \sum_{r=1}^{\infty} \frac{C}{r^{\gamma \alpha / 2}}\left(\mathbf{E}\left(\frac{\left\|X_{1}\right\|^{2}}{L L\left\|X_{1}\right\|} I\left(\left\|X_{1}\right\|^{2} \leqslant \tau_{r}\right) \frac{L L\left\|X_{1}\right\|}{L L \tau_{r}}\right)\right)^{(2+\alpha) / 2}
\end{aligned}
$$

Из (11) вытекает сходимость последнего ряда.

Теперь оценим $I$ (везде ниже $\phi(x)=\log _{\beta} x$ ):

$$
\begin{aligned}
I & =\sum_{r=1}^{\infty} \sum_{i=1}^{m\left(\left[\beta^{r+1}\right]\right)} \frac{C}{\alpha_{n_{r+1}}^{2+\alpha}} \sum_{j=(p+q)(i-1)+1}^{(p+q)(i-1)+p} \mathbf{E} \| X_{j} I\left(\left\|X_{j}\right\|^{2} \leqslant \tau_{r}\right) \\
& \leqslant \sum_{r=1}^{\infty} \frac{C \beta^{r+1} p}{p \alpha_{n_{r+1}}^{2+\alpha}} \mathbf{E}\left(\left\|X_{1}\right\|^{2+\alpha} I\left(\left\|X_{1}\right\|^{2} \leqslant \tau_{r}\right)\right) \\
& \leqslant \sum_{r=1}^{\infty} \frac{C \beta^{r+1}}{\left.\alpha_{n_{r+1}}^{2+\alpha} \|^{2} \leqslant \tau_{r}\right) \|^{2+\alpha} \mathbf{E}\left(\left\|X_{1}\right\|^{2+\alpha} I\left(\left\|X_{1}\right\|^{2} \leqslant \tau_{r}\right)\right)} \\
& \leqslant C \sum_{r=1}^{\infty} \sum_{k=1}^{\alpha_{n}+1} \frac{k^{(2+\alpha) / 2} \mathbf{P}\left\{k-1 \leqslant\left\|X_{1}\right\|^{2} \leqslant k\right\}}{\beta^{r \alpha / 2}\left(L L \beta^{r}\right)^{(2+\alpha) / 2}} \\
& \leqslant C \sum_{k=1}^{\infty} \frac{k^{(2+\alpha) / 2} \mathbf{P}\left\{k-1 \leqslant\left\|X_{1}\right\|^{2} \leqslant k\right\}}{\beta^{r \alpha / 2}\left(L L \beta^{r}\right)^{(2+\alpha) / 2}} \\
& \leqslant C \sum_{k=1}^{\infty} \frac{k^{(2+\alpha) / 2} \mathbf{P}\left\{k-1 \leqslant\left\|X_{1}\right\|^{2} \leqslant k\right\}}{(k /(L L k))^{\alpha / 2}(L L(k /(L L k)))^{(2+\alpha) / 2}} \\
& \leqslant C \sum_{k=1}^{\infty} \frac{k}{L L k} \mathbf{P}\left\{k-1 \leqslant\left\|X_{1}\right\|^{2} \leqslant k\right\} \leqslant C \mathbf{E} \frac{\left\|X_{1}\right\|^{2}}{L L\left\|X_{1}\right\|}<\infty
\end{aligned}
$$


Нам остается доказать, что

$$
\sum_{k=1}^{\infty} \mathbf{E} g\left(\sum_{i=1}^{m\left(\left[\beta^{r+1}\right]\right)} \frac{G_{i}}{\alpha_{n_{r+1}}}\right)<\infty .
$$

Имеем

$$
\begin{aligned}
\mathbf{E} g\left(\sum_{i=1}^{m\left(\left[\beta^{r+1}\right]\right)} \frac{G_{i}}{\alpha_{n_{r+1}}}\right) & \leqslant \mathbf{E} I\left(\left\|\sum_{i=1}^{m\left(\left[\beta^{r+1}\right]\right)} \frac{G_{i}}{\alpha_{n_{r+1}}}\right\|>\Gamma+\frac{\varepsilon}{8}\right) \\
& =\mathbf{P}\left\{\left\|\sum_{i=1}^{m\left(\left[\beta^{r+1}\right]\right)} \frac{G_{i}}{\alpha_{n_{r+1}}}\right\|>\Gamma+\frac{\varepsilon}{8}\right\} .
\end{aligned}
$$

Покажем, что

$$
\sum_{k=1}^{\infty} \mathbf{P}\left\{\left\|\sum_{i=1}^{m\left(\left[\beta^{r+1}\right]\right)} \frac{G_{i}}{\alpha_{n_{r+1}}}\right\|>\Gamma+\frac{\varepsilon}{8}\right\} .
$$

Воспользуемся следующей леммой.

Лемма 6 (см. [2]). Пусть $G$ - гауссовская с.в. в В с нулевым средним $и$

$$
\Gamma_{G}=\sup _{x \in K_{G}}\|x\|
$$

әде $K_{G}$ получено также, как в лемме 1 , по распределению с.в. G. Для фиксированных $t_{0}>0, \alpha>0$ обозначим

$$
\begin{gathered}
\lambda(G)=\lambda_{t_{0}, \alpha}(G)=\inf \left\{\lambda>0: \mathbf{P}\left\{\frac{\|G\|}{\lambda}<t_{0}\right\} \geqslant F(\alpha)\right\} \\
F(x)=\int_{-\infty}^{x} \frac{e^{-u^{2} / 2}}{\sqrt{2 \pi}} d u .
\end{gathered}
$$

Тогда для всех $t \geqslant t_{0}$ u $\rho>\lambda(G)$

$$
\mathbf{P}\{\|G\| \geqslant \rho t\} \leqslant 1-F\left(\alpha+\Gamma_{G}^{-1} \rho\left(t-t_{0}\right)\right) .
$$

Положим

$$
G=\sum_{i=1}^{m\left(\left[\beta^{r+1}\right]\right)} \frac{G_{i}}{\alpha_{n_{r+1}}}, \quad \Gamma_{G}=\Gamma_{r}
$$

и покажем, что $\mathbf{E}\|G\|^{2}=o\left(L L n_{r+1}\right)$ :

$$
\begin{aligned}
& \lim _{r \rightarrow \infty} \mathbf{E}\left\|\sum_{i=1}^{m\left(\left[\beta^{r+1}\right]\right)} \frac{G_{i}}{\alpha_{n_{r+1}}}\right\|^{2} \leqslant \lim _{r \rightarrow \infty} \frac{1}{\alpha_{n_{r+1}}^{2}} \sum_{i=1}^{m\left(\left[\beta^{r+1}\right]\right)} \mathbf{E}\left\|G_{i}\right\|^{2} \\
& \quad \leqslant \lim _{r \rightarrow \infty} \frac{1}{\alpha_{n_{r+1}}^{2}} \sum_{i=1}^{m\left(\left[\beta^{r+1}\right]\right)} \mathbf{E}\left\|\xi_{i}\right\|^{2} \leqslant \lim _{r \rightarrow \infty} \frac{C \beta^{r+1} p}{p \alpha_{n_{r+1}}^{2}} \mathbf{E}\left\|X_{1}\right\|^{2} I\left(\left\|X_{1}\right\|^{2} \leqslant \tau_{r}\right) \\
& \leqslant C \lim _{r \rightarrow \infty} \mathbf{E}\left(\frac{\left\|X_{1}\right\|^{2} I\left(\left\|X_{1}\right\|^{2} \leqslant \tau_{r}\right)}{L L n_{r+1}}\right) \\
& \leqslant C \lim _{r \rightarrow \infty} \mathbf{E}\left(\frac{\left\|X_{1}\right\|^{2}}{L L\left\|X_{1}\right\|} I\left(\left\|X_{1}\right\|^{2} \leqslant \tau_{r}\right) \frac{L L\left\|X_{1}\right\|}{L L n_{r+1}}\right)=0 .
\end{aligned}
$$


Выше мы использовали следующий факт о сходимости вторых моментов в центральной предельной теореме (см. [26]):

$$
\mathbf{E}\left\|G_{i}\right\|^{2}=\lim _{r \rightarrow \infty} \mathbf{E}\left\|n^{-1 / 2}\left(y_{i 1}+\cdots+y_{i n}\right)\right\|^{2}=\mathbf{E}\left\|y_{i 1}\right\|^{2}=\mathbf{E}\left\|\xi_{i}\right\|^{2},
$$

где $\left\{y_{i k} ; k \geqslant 1\right\}$ - независимые копии $y_{i}$. Из соотношения $\mathbf{E}\|G\|^{2}=$ $o\left(L L n_{r+1}\right)$ и неравенства Чебышева следует, что для фиксированных $t_{0}>0, \alpha>0$

$$
\lambda(G)=o\left(\left(L L n_{r+1}\right)^{1 / 2}\right) \quad \text { при } \quad r \rightarrow \infty .
$$

Таким образом, для любого $\theta>0$ и достаточно больших $r$

$$
\lambda(G)=\theta\left(L L n_{r+1}\right)^{1 / 2}\left(\Gamma+\frac{\varepsilon}{8}\right) .
$$

Используя лемму 6 , для любого фиксированного $\theta$ и $r \geqslant r_{0}(\theta)$ при $t>t_{0}$ имеем

$$
\begin{aligned}
& \mathbf{P}\left\{\frac{\|G\|}{\theta\left(L L n_{r+1}\right)^{1 / 2}(\Gamma+\varepsilon / 8)}>t\right\} \\
& \quad \leqslant 1-F\left(\alpha+\Gamma_{r}^{-1} \theta\left(L L n_{r+1}\right)^{1 / 2}\left(\Gamma+\frac{\varepsilon}{8}\right)\left(t-t_{0}\right)\right) .
\end{aligned}
$$

Покажем, что $\Gamma_{r} \rightarrow \Gamma$ при $r \rightarrow \infty$. Напомним, что

$$
\begin{aligned}
& \Gamma_{r}=\left(\sup _{\|f\| \leqslant 1} \mathbf{E} f^{2}(G)\right)^{1 / 2}=\sup _{\|f\| \leqslant 1}\left(\mathbf{E} f^{2}\left(\sum_{i=1}^{m\left(\left[\beta^{r+1}\right]\right)} \frac{G_{i}}{\sqrt{n_{r+1}}}\right)\right)^{1 / 2}, \\
& \Gamma=\sup _{\|f\| \leqslant 1}(T(f, f))^{1 / 2}=\sup _{\|f\| \leqslant 1} \lim _{n \rightarrow \infty}\left(\frac{\mathbf{E} f^{2}\left(S_{n}\right)}{n}\right)^{1 / 2} .
\end{aligned}
$$

Докажем следующее соотношение:

$$
\left|\mathbf{E} f^{2}\left(\sum_{i=1}^{m\left(\left[\beta^{r+1}\right]\right)} \frac{G_{i}}{\sqrt{n_{r+1}}}\right)-\frac{\mathbf{E} f^{2}\left(S_{n_{r+1}}\right)}{n_{r+1}}\right| \longrightarrow 0 \quad \text { при } \quad r \rightarrow \infty .
$$

В силу независимости $\left\{G_{i}\right\}$ и того факта, что $G_{i}$ и $y_{i}$ имеют одинаковый ковариационный оператор, получим

$$
\begin{aligned}
\mathbf{E} f^{2}\left(\sum_{i=1}^{m\left(\left[\beta^{r+1}\right]\right)} \frac{G_{i}}{\sqrt{n_{r+1}}}\right) & =\frac{1}{n_{r+1}} \sum_{i=1}^{m\left(\left[\beta^{r+1}\right]\right)} \mathbf{E} f^{2}\left(G_{i}\right)=\frac{1}{n_{r+1}} \sum_{i=1}^{m\left(\left[\beta^{r+1}\right]\right)} \mathbf{E} f^{2}\left(y_{i}\right) \\
& =\frac{1}{n_{r+1}} \sum_{i=1}^{m\left(\left[\beta^{r+1}\right]\right)} \mathbf{E} f^{2}\left(\xi_{i}\right) .
\end{aligned}
$$

Теперь докажем, что при $r \rightarrow \infty$

$$
\left|\frac{1}{n_{r+1}} \sum_{i=1}^{m\left(\left[\beta^{r+1}\right]\right)} \mathbf{E} f^{2}\left(\xi_{i}\right)-\frac{1}{n_{r+1}} \mathbf{E} f^{2}\left(\sum_{i=1}^{m\left(\left[\beta^{r+1}\right]\right)} \xi_{i}\right)\right| \longrightarrow 0 .
$$

Используем следующую лемму. 
Лемма 7 (см. [23]). Для иентрированной последовательности с.в. $\left\{X_{i}, i \geqslant 1\right\}$ со значениями в гильбертовом пространстве имеет место следуюшее неравенство:

$$
\begin{aligned}
& \left|\sum_{i, j=1,|i-j|>1}^{n} \mathbf{E}\left\langle X_{i}, X_{j}\right\rangle\right| \\
& \leqslant C \exp \left(2 \sum_{j=0}^{\left[\log _{2} n\right]} \psi^{1 / 2}\left(2^{j / 3}\right)\right) \sum_{j=0}^{\left[\log _{2} n\right]} \psi^{1 / 2}\left(2^{j / 3}\right) \sum_{j=1}^{n} \mathbf{E}\left\|X_{j}\right\|^{2} .
\end{aligned}
$$

Здесь $C$ - абсолютная константа, $\langle\cdot, \cdot\rangle$ - скалярное произведение.

Используя эту лемму для одномерных с.в. $f\left(\xi_{i}\right)$, а также лемму 3 и имея в виду (3), получим:

$$
\begin{aligned}
& \left|\frac{1}{r_{i r+1}}\left(\sum_{i=1}^{m\left(\left[\beta^{r+1}\right]\right)} \mathbf{E} f^{2}\left(\xi_{i}\right)-\mathbf{E} f^{2}\left(\sum_{i=1}^{m\left(\left[\beta^{r+1}\right]\right)} \xi_{i}\right)\right)\right| \\
& =\left|\frac{1}{n_{r+1}} \sum_{i, j=1,|i-j|>1}^{m\left(\left[\beta^{r+1}\right\}\right)} \mathbf{E} f\left(\xi_{i}\right) f\left(\xi_{j}\right)\right| \\
& \leqslant \frac{1}{n_{r+1}} \exp \left(2 C \frac{\log _{2} r}{r^{2}}\right) \frac{\log _{2} r}{r^{2}} \sum_{i=1}^{m\left(\left[\beta^{r+1}\right]\right)} \mathbf{E} f^{2}\left(\xi_{i}\right) \\
& \leqslant \frac{C \exp \left(2 C\left(\log _{2} r\right) / r^{2}\right) \log _{2} r}{\beta^{2} r^{2}} \sum_{i=1}^{m\left(\left[\beta^{r+1}\right]\right)} \mathbf{E} f^{2}\left(\sum_{j=(p+q)(i-1)+1}^{(p+q)(i-1)+p} u_{j}\right) \\
& \leqslant \frac{C \exp \left(2 C\left(\log _{2} r\right) / r^{2}\right) \log _{2} r}{\beta^{2} r^{2}} r^{\gamma} p \mathbf{E} f^{2}\left(u_{(p+q)\left(m\left(\left[\beta^{r+1}\right]\right)-1\right)+p}\right) \\
& \leqslant \frac{C \log _{2} r}{\beta^{2} r^{2}} \mathbf{E} f^{2}\left(u_{(p+q)\left(m\left(\left[\beta^{r+1}\right]\right)-1\right)+p}\right) \longrightarrow 0 \quad \text { при } \quad r \rightarrow \infty .
\end{aligned}
$$

Теперь покажем, что при $r \rightarrow \infty$

$$
\frac{1}{n_{r+1}}\left|\mathbf{E} f^{2}\left(\sum_{i=1}^{m\left(\left[\beta^{r+1}\right]\right)} \xi_{i}\right)-\mathbf{E} f^{2}\left(\sum_{i=1}^{m\left(\left[\beta^{r+1}\right]\right)} u_{i}\right)\right| \longrightarrow 0 .
$$

Имеем

$$
\begin{aligned}
\mid \frac{1}{n_{r+1}} & \left(\mathbf{E} f^{2}\left(\sum_{i=1}^{m\left(\left[\beta^{r+1}\right]\right)} \xi_{i}\right)-\mathbf{E} f^{2}\left(\sum_{i=1}^{m\left(\left[\beta^{r+1}\right]\right)} u_{i}\right)\right) \mid \\
= & \frac{1}{n_{r+1}}\left(\mathbf{E} f^{2}\left(\sum_{i=1}^{m\left(\left[\beta^{r+1}\right]\right)} \xi_{i}\right)-\mathbf{E} f^{2}\left(\sum_{i=1}^{m\left(\left[\beta^{r+1}\right]\right)} \xi_{i}+\sum_{i=1}^{k} \eta_{i}+U_{0}\right)\right) \mid \\
= & \frac{1}{n_{r+1}}\left(\mathbf{E} f^{2}\left(\sum_{i=1}^{k} \eta_{i}\right)+\mathbf{E} f^{2}\left(U_{0}\right)+2 \mathbf{E} f\left(\sum_{i=1}^{m\left(\left[\beta^{r+1}\right]\right)} \xi_{i}\right) f\left(\sum_{i=1}^{k} \eta_{i}\right)\right. \\
& \left.+2 \mathbf{E} f\left(\sum_{i=1}^{m\left(\left[\beta^{r+1}\right]\right)} \xi_{i}\right) f\left(U_{0}\right)+2 \mathbf{E} f\left(\sum_{i=1}^{k} \eta_{i}\right) f\left(U_{0}\right)\right) \mid .
\end{aligned}
$$


Сходимость последнего выражения к нулю при $r \rightarrow \infty$ вытекает из следующих пяти соотношений:

$$
\begin{aligned}
& \frac{1}{n_{r+1}} \mathbf{E} f^{2}\left(\sum_{i=1}^{k} \eta_{i}\right) \leqslant \frac{C k q}{n_{r+1}} \mathbf{E} f^{2}\left(u_{(p+q) k}\right) \\
& \leqslant \frac{C r^{\gamma} \beta^{\alpha_{0} r}}{\beta^{r+1}} \mathbf{E} f^{2}\left(u_{(p+q) k}\right) \longrightarrow 0 ; \\
& \frac{1}{n_{r+1}} \mathbf{E} f^{2}\left(U_{0}\right) \leqslant \frac{C p}{n_{r+1}} \mathbf{E} f^{2}\left(u_{n_{r+1}}\right) \\
& \leqslant \frac{C \frac{\beta^{r}}{r^{\gamma}}}{n_{r+1}} \mathbf{E} f^{2}\left(u_{n_{r+1}}\right) \longrightarrow 0 ; \\
& \left|\frac{2}{n_{r+1}} \mathbf{E} f\left(\sum_{i=1}^{m\left(\left[\beta^{r+1}\right]\right)} \xi_{i}\right) f\left(\sum_{i=1}^{k} \eta_{i}\right)\right| \\
& \leqslant \frac{2}{n_{r+1}}\left(\mathbf{E} f^{2}\left(\sum_{i=1}^{m\left(\left[\beta^{r+1}\right]\right)} \xi_{i}\right)\right)^{1 / 2}\left(\mathbf{E} f^{2}\left(\sum_{i=1}^{k} \eta_{i}\right)\right)^{1 / 2} \\
& \leqslant \frac{C \sqrt{m\left(\left[\beta^{r+1}\right]\right) p} \sqrt{k q}}{n_{r+1}} \leqslant \frac{C \sqrt{r^{\gamma}\left(\beta^{r} / r^{\gamma}\right) r^{\gamma} \beta^{r \alpha_{0}}}}{\beta^{r+1}} \\
& \leqslant \frac{C \beta^{\left(1 / 2+\alpha_{0} / 2\right) r}}{\beta^{r}} \longrightarrow 0 ; \\
& \left|\frac{2}{n_{r+1}} \mathbf{E} f\left(\sum_{i=1}^{m\left(\left[\beta^{r+1}\right]\right)} \xi_{i}\right) f\left(U_{0}\right)\right| \leqslant \frac{2}{n_{r+1}}\left(\mathbf{E} f^{2}\left(\sum_{i=1}^{k} \xi_{i}\right)\right)^{1 / 2}\left(\mathbf{E} f^{2}\left(U_{0}\right)\right)^{1 / 2} \\
& \leqslant \frac{C \sqrt{r^{\gamma}\left(\beta^{r} / r^{\gamma}\right)} \sqrt{\beta^{r} / r^{\gamma}}}{\beta^{r}} \leqslant \frac{C}{r^{\gamma / 2}} \longrightarrow 0 ; \\
& \left|\frac{2}{n_{r+1}} \mathbf{E} f\left(\sum_{i=1}^{k} \eta_{i}\right) f\left(U_{0}\right)\right| \leqslant \frac{2}{n_{r+1}}\left(\mathbf{E} f^{2}\left(\sum_{i=1}^{k} \eta_{i}\right)\right)^{1 / 2}\left(\mathbf{E} f^{2}\left(U_{0}\right)\right)^{1 / 2} \\
& \leqslant \frac{C \sqrt{r^{\gamma} \beta^{\alpha_{0} r}} \sqrt{\beta^{r} / r^{\gamma}}}{\beta^{r}} \leqslant \frac{C \beta^{\left(\alpha_{0} / 2+1 / 2\right) r}}{\beta^{r}} \longrightarrow 0 .
\end{aligned}
$$

Наконец, остается доказать, что при $r \rightarrow \infty$

$$
\left|\frac{1}{n_{r+1}}\left(\mathbf{E} f^{2}\left(\sum_{i=1}^{n_{r+1}} \eta_{i}\right)-\mathbf{E} f^{2}\left(S_{n_{r+1}}\right)\right)\right| \rightarrow 0 .
$$

Имеем

$$
\begin{aligned}
& \left|\frac{1}{n_{r+1}}\left(\mathbf{E} f^{2}\left(\sum_{i=1}^{n_{r+1}} \eta_{i}\right)-\mathbf{E} f^{2}\left(S_{n_{r+1}}\right)\right)\right| \\
& \quad=\left|\frac{1}{n_{r+1}}\left(\mathbf{E} f^{2}\left(\sum_{i=1}^{n_{r+1}} \eta_{i}\right)-\mathbf{E} f^{2}\left(U_{n_{r+1}}+W_{n_{r+1}}\right)\right)\right| \\
& \quad=\frac{1}{n_{r+1}}\left|\mathbf{E} f^{2}\left(W_{n_{r+1}}\right)+\mathbf{E} f\left(U_{n_{r+1}}\right) f\left(W_{i}\right)\right| .
\end{aligned}
$$


Далее, из леммы 3 получаем

$$
\frac{1}{n_{r+1}} \mathbf{E} f^{2}\left(W_{n_{r+1}}\right) \leqslant C \frac{\sum_{i=1}^{n_{r+1}} \mathbf{E} f^{2}\left(w_{i}\right)}{n_{r+1}}
$$

Из соотношения $\mathbf{E} f^{2}\left(w_{n_{r+1}}\right) \rightarrow 0$ при $r \rightarrow \infty$ и известной леммы Тёплица вытекает, что

$$
\frac{\sum_{i=1}^{n_{r+1}} \mathbf{E} f^{2}\left(w_{n_{r+1}}\right)}{n_{r+1}} \longrightarrow 0
$$

при $r \rightarrow \infty$. Далее,

$$
\begin{gathered}
\frac{1}{n_{r+1}}\left|\mathbf{E} f\left(U_{n_{r+1}}\right) f\left(W_{n_{r+1}}\right)\right| \leqslant \frac{1}{n_{r+1}}\left(\mathbf{E} f^{2}\left(U_{n_{r+1}}\right)\right)^{1 / 2}\left(\mathbf{E} f^{2}\left(W_{n_{r+1}}\right)\right)^{1 / 2} \\
\leqslant \frac{C \sqrt{n_{r+1}}\left(\mathbf{E} f^{2}\left(W_{n_{r+1}}\right)\right)^{1 / 2}}{n_{r+1}} \leqslant C\left(\frac{\mathbf{E} f^{2}\left(W_{n_{r+1}}\right)}{n_{r+1}}\right)^{1 / 2} .
\end{gathered}
$$

Сходимость к нулю последнего выражения была уже нами доказана.

Остается выбрать такое достаточно малое $\theta>0$, чтобы $t=\theta^{-1}$ было больше $t_{0}$ и для достаточно больших $r$ выполнялось неравенство

$$
\Gamma_{r}^{-1} \theta\left(\Gamma+\frac{\varepsilon}{8}\right)\left(\frac{1}{\theta}-t_{0}\right)>1+\delta \quad \text { для некоторого } \delta>0 .
$$

При таком выборе $\theta$ и $t=\theta^{-1}$ для достаточно больших $r$ имеем

$$
\begin{aligned}
\mathbf{P}\left\{\|G\|>\left(L L n_{r+1}\right)^{1 / 2}\left(\Gamma+\frac{\varepsilon}{8}\right)\right\} & \leqslant 1-F\left((1+\delta)\left(L L n_{r+1}\right)^{1 / 2}\right) \\
& \leqslant \exp \left\{-\left(1+\frac{\delta}{2}\right) L L n_{r+1}\right\},
\end{aligned}
$$

откуда в свою очередь следует (18). Таким образом, мы доказали (15). Доказательство достаточности закончено.

$\mathrm{H}$ е о 6 х о д и м о с т ь. Сначала приведем закон «0 или $1 \gg$ для последовательности с.в. с сильным перемешиванием.

Напомним, что коэффициент сильного перемешивания для последовательности $\left\{X_{n}, n \geqslant 1\right\}$ определяется следующим образом:

$$
\alpha(k)=\sup \left\{|\mathbf{P}(A B)-\mathbf{P}(A) \mathbf{P}(B)|: A \in \mathscr{F}_{1}^{n}, B \in \mathscr{F}_{k+n}^{\infty}, n \in \mathbf{N}\right\},
$$

где $\mathscr{F}_{k}^{n}-\sigma$-алгебра, порожденная с.в. $X_{k}, \ldots, X_{n}$.

Лемма 8. Пусть $\left\{X_{n}, n \geqslant 1\right\}$ последовательность с.в. со значениями в любом сепарабельном банаховом пространстве, которая удовлетворяет следуюшему условию: $\lim _{k \rightarrow \infty} \alpha(k)=0$. Тогда «хвостовая» $\sigma$-алгебра $\mathscr{F}=\bigcap_{n=1}^{\infty} \mathscr{F}_{n}^{\infty}$ тривиальна, т.е. для любого $A \in \mathscr{F}$ имеем $\mathbf{P}(A)=0$ или $\mathbf{P}(A)=1$.

Для полноты изложения приведем доказательство леммы 8 (см. также [27]). Мы будем следовать доказательству теоремы 1 из [28, с. 368]. Там доказано, что для любого $A \in \mathscr{F}$ существуют множества $A_{n} \in \mathscr{F}_{1}^{n}$, 
$n \geqslant 1$, такие, что $\mathbf{P}\left(A_{n} A\right) \rightarrow \mathbf{P}(A), \mathbf{P}\left(A_{n}\right) \rightarrow \mathbf{P}(A)$ при $n \rightarrow \infty$, что доказано в [28, с. 368] (см. доказательство теоремы 1).

Так как $A \in \mathscr{F}$ то $A \in \mathscr{F}_{n+k}^{\infty}$ для любого $k \geqslant 1$. Из определения коэффициента перемешивания получим

$$
\left|\mathbf{P}\left(A_{n} A\right)-\mathbf{P}\left(A_{n}\right) \mathbf{P}(A)\right| \leqslant \alpha(k) \quad \text { для любого } k \geqslant 1,
$$

что вместе с условием леммы 8 влечет $\mathbf{P}(A)=\mathbf{P}^{2}(A)$. Откуда и следует утверждение леммы 8.

Лемма 8 и соотношение (1) влекут сушествование постоянной $c$ такой, что $\lim \sup _{n \rightarrow \infty}\left(\left\|S_{n}\right\| / \alpha_{n}\right)=c$ п.н., откуда вытекает существование константы $K$ такой, что

$$
\mathbf{P}\left\{\left\|\frac{X_{n}}{\alpha_{n}}\right\|>K \text { бесконечно часто }\right\}=0 .
$$

Используем следующую лемму, которую сформулируем в нужной нам форме.

Лемма 9 (см. [29]). Для последовательности $\left\{X_{n}, n \geqslant 1\right\}$ с.в., удовлетворяюших условию $\psi$-перемешивания и принимаюших значения в $B$, условия (24) достаточно для того, чтоби $\sum_{n=1}^{\infty} \mathbf{P}\left\{\left\|X_{n} / \alpha_{n}\right\|>\right.$ $K\}<\infty$.

Отсюда получаем, что

$$
\sum_{n=1}^{\infty} \mathbf{P}\left\{\left\|\frac{X_{n}}{\alpha_{n}}\right\|>K\right\}=\sum_{n=1}^{\infty} \mathbf{P}\left\{\left\|X_{1}\right\|>K \alpha_{n}\right\}<\infty .
$$

Сходимость последнего ряда эквивалентна условию (5).

Таким образом, доказательство теоремы закончено.

Автор приносит свою благодарность рецензенту за полезные замечания и предложения, способствовавшие улучшению первоначального варианта статьи.

\section{СПИСОК ЛИТЕРАТУРЫ}

1. Goodman V., Kuelbs J., Zinn J. Some results on the LIL in Banach space with applications to weighted empirical processes. - Ann. Probab., 1981, v. 9, № 5, p. 713752 .

2. de Acosta A., Kuelbs J. Some results on the cluster sets $C\left(\left\{S_{n} / a_{n}\right\}\right)$ and the LIL. Ann. Probab., 1983, v. 11, № 1, p. 102-122.

3. Ledoux $M$., Talagrand $M$. Characterization of the law of the iterated logarithm in Banach spaces. - Ann. Probab., 1988, v. 16, № 3, p. 1242-1264.

4. Kuelbs J. A strong convergence theorem for Banach space valued random variables. Ann. Probab., 1976, v. 4, № 5, p. 744-771.

5. Kuelbs J., Philipp W. Almost sure invariance principles for partial sums of mixing $B$-valued random variables. - Ann. Probab., 1980, v. 8, №6, p. 1003-1036.

6. Dehling H., Phillipp W. Almost sure invariance principles for weakly dependent vector-valued random variables. - Ann. Probab., 1982, v. 10, № 3, p. 689-701.

7. Berkes I., Philipp W. Approximation theorems for independent and weakly dependent random vectors. - Ann. Prcbab., 1979, v. 7, № 1, p. 29-54. 
8. Phillipp $W$. Almost sure invariance principles for sums of $B$-valued random variables. - Lecture Notes in Math., 1979, v. 709, p. 171-193.

9. Berger $E$. An almost sure invariance principles for stationary ergodic sequences of Banach space valued random variables. - Probab. Theory Related. Fields, 1990, v. 84 , p. $161-201$.

10. Einmahl U. Stability results and strong invariance principles for partial sums of Banach space valued random variables. - Ann. Probab., 1989, v. 17, № 1, p. 333-352.

11. Li D. L., Rao M. B., Wang X. C. The law of the iterated logarithm for independent random variables with multidimensional indices. - Ann. Probab., 1992, v. 20, № 2, p. $660-674$.

12. Chen $X$. On the law of the iterated logarithm for independent Banach space valued random variables. - Ann. Probab., 1993, v. 21, № 4, p. 1991-2011.

13. Einmahl $U$. Toward a general law of the iterated logarithm in Banach space. - Ann. Probab., 1993, v. 21, № 4, p. 2012-2045.

14. Chen X. On Strassen's law of the iterated logarithm in Banach space. - Ann. Probab., 1994 , v. 22 , № 2 , p. 1026-1043.

15. Einmahl $U$. On the cluster set problem for the generalized law of the iterated logarithm in Euclidean space. - Ann. Probab., 1995, v. 23, № 2, p. 817-851.

16. Li D., Tomkins R.J. Compact laws of the iterated logarithm for B-valued random variables with two dimensional indices. - J. Theoret. Probab., 1998, v. 11, № 2, p. $443-459$.

17. Шарипов О.Ш. Предельные теоремы для слабозависимых случайных величин со значениями в пространстве $l_{p}$. Автореферат дисс... .канд. физ.-матем. наук, 1993, Институт математики им. В. И. Романовского АН Узбекистана, Ташкент, $16 \mathrm{c}$.

18. Chen $X$. The law of the iterated logarithm for $m$-dependent Banach space valued random variables. - J. Theoret. Probab., 1997, v. 10, № 3, p. 695-732.

19. Kuelbs $J$. An inequality for the distribution of a sum of certain Banach space valued random variables. - Studia Math., 1974, v. 52, p. 69-87.

20. Peligrad $M$. Invariance principles for mixing sequences of random variables. - Ann. Probab., 1982, v. 10, № 4, p. 968-981.

21. Шарипов О.ШI. Закон повторного логарифма для слабо зависимых случайных величин со значениями в гильбертовом пространстве. - Сиб. матем. журнал, 2003 , т. 44 , № 6 , c. $1407-1424$.

22. Sharipov O.Sh. Strong limit theorems for mixing random variables with values in Hilbert space and their applications. - Progress in Probability. Ed. by M. Marcus and J. Wellner. Birkhauser-Verlag-Basel: Switzerland, 2003, v. 55, p. 153-174.

23. Утев С. А. Суммы случайных величин с $\phi$-перемешиванием. - Труды Ин-та Математики СО АН РАН, 1989, т. 13, с. 78-100.

24. Шарипов О. Ш. О вероятностной характеризации банаховых пространств слабозависимыми случайными элементами. - Узб. матем. журн., 1998, № 2, с. 86-91.

25. Iosifescu M., Theodorescu R. Random Processes and Learning. New York: SpringerVerlag, 1969, $304 \mathrm{p}$.

26. de Acosta A., Giné E. Convergence of moments and related functionals in the general central limit theorem in Banach spaces. - Z. Wahrscheinlichkeitstheor. Verw. Geb., 1979 , v. 48, № 2 , p. 213-231.

27. Bradley R. C. Introduction to strong mixing conditions, v. I. Technical report, Department of Mathematics, Indiana University, Bloomington. Custom Publishing of I. U., Bloomington, March, 2002, 528 p.

28. Ширяев А.Н. Вероятность. М.: Наука, 1980, 576 с.

29. Cohn $H$. On a class of dependent random variables. - Rev. Roumaine Math. Pures Appl., 1965, v. 10, p.1593-1606. 